\title{
Motivation System of Crowdsourcing Community from a Supply Chain Perspective
}

\author{
Jiangang Pang ${ }^{1,2}$ and Zhiying Liu ${ }^{2}$ \\ ${ }^{1}$ Southwest University of Science and Technology, Mianyang, Sichuan 621010, China \\ ${ }^{2}$ University of Science and Technology of China, Hefei, Anhui 230026, China \\ Correspondence should be addressed to Jiangang Pang; 392210825@qq.com
}

Received 7 December 2015; Accepted 9 June 2016

Academic Editor: Zhimin Huang

Copyright (C) 2016 J. Pang and Z. Liu. This is an open access article distributed under the Creative Commons Attribution License, which permits unrestricted use, distribution, and reproduction in any medium, provided the original work is properly cited.

\begin{abstract}
This paper uses principal-agent theory to study the issue of incentivizing crowdsourcing communities. It proves that enterprises can generate innovation plans of high quality and expected utility using the crowdsourcing community. Outsourcers can encourage high-quality people to join by adopting a linear variable compensation scheme and make the low-quality people quit by requiring them to supply more effort. The paper also shows that enterprises' participation in crowdsourcing community innovation can effectively improve their innovative ability and that it is necessary for enterprises to construct an effective and cooperative innovative system combining crowdsourcing community innovation and their own internal innovation.
\end{abstract}

\section{Introduction}

Rising competition forces enterprises to continuously explore ways to improve production. Furthermore, the development of the Internet has offered enterprises tools that encourage innovation more broadly (open innovation based on the Internet is very popular in many enterprises; e.g., IBM built its own innovation platform, Innovation Jam, in 2001; Dell built the Dell Online Community covering the functions of ideastom, directdell, dellforums, and studiodell in 2002; InnoCentive, the global third-party Internet innovative platform, built in 2001, appealed to the world's top 500 companies). In 2006, Howe defined the cooperative innovative model of mass participation based on the Internet as crowdsourcing [1], wherein a company or an organization subcontracts assignments normally done by internal staff to online contractors. These assignments can include the development of new technology, completeness of a new design, improvement of a calculation method, and analysis of mass data, which are performed by crowdsourcing communities such as Innovation Jam, Dell Online Community, InnoCentive, Starbucks, and Witkey. For such a crowdsourcing model to be successful, it is imperative that online contractors find it in their best interest to participate. Therefore, it is important to design a motivation system for the crowdsourcing community that effectively increases the initiative of participants and thereby enhance the quality of the output.

This paper analyzes incentive mechanisms on community-based crowdsourcing and studies how to best carry out the design of these incentive mechanisms. Through the analysis of the enterprise, the community-based crowdsourcing business innovation scheme can obtain higher quality and can bring about higher expected utility for enterprises. Contract-issuing party that participates in the crowdsourcing community process, using linear variable compensation incentive strategy, can get higher level network public participation and at the same time can generate general technical level network public participation but it needs to give up on putting a lot of effort on it and better enhance the innovation quality, putting forward linear crowdsourcing community innovation incentive mechanism design. Based on the conclusions, putting forward enterprises to participate in the crowdsourcing community innovation can be more effective than enhancing the innovation ability of the enterprises; at the same time, the enterprises should establish innovation system based on crowdsourcing community innovation and 


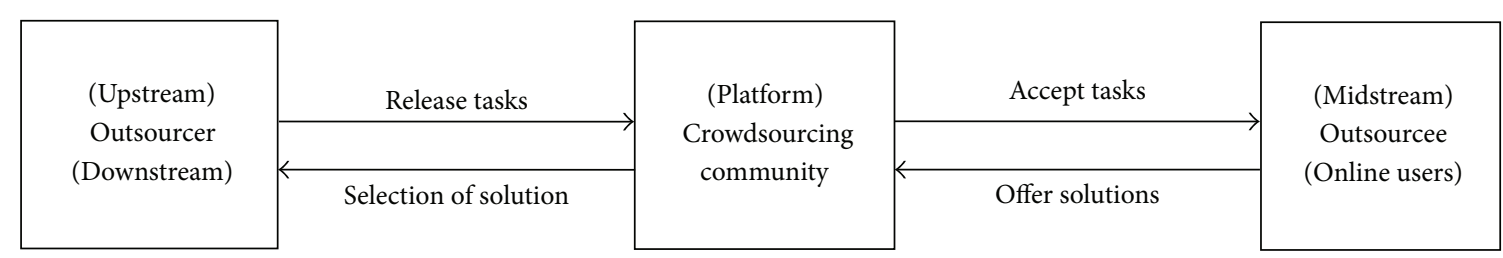

FIGURE 1: Crowdsourcing community supply chain.

enterprise innovation effective coordination. Before proceeding further, we define and clarify the jargon we use in this paper.

1.1. Crowdsourcing Community Participants. In existing crowdsourcing communities, the participants include outsourcers, the enterprises that need outsourcing services, and outsourcees, the solvers of problems (solvers of problems: in crowdsourcing community, participants mainly include outsourcer (enterprises in need), crowdsourcing community (crowdsourcing platform), and outsourcee (problem solvers, principally, are a mass participating crowdsourcing community); the solvers of problems are the main participant).

Outsourcers. The outsourcers are the enterprises or individuals that need the outsourcing service. There are two ways in which the outsourcer releases tasks. One way is by building a crowdsourcing community to attract outsourcees to solve problems by offering rewards. The other way is to partner with a third-party crowdsourcing community, such as help.zhubajie.com and InnoCentive.

Outsourcees. The outsourcee refers to crowdsourcing users who could be both professionals and nonprofessionals or teams who have an interest in and capability of studying tasks from all over the world. They can register via the third-party crowdsourcing community and accept such agreements as the selection of superior solutions, the fulfillment of offering rewards, and disposal methods of intellectual property and then serve as problem solvers who execute the activities of solving problems and submit solutions through the crowdsourcing community.

Crowdsourcing Community. A crowdsourcing community is the platform that connects outsourcers and outsourcees by developing either a crowdsourcing community (e.g., Amazon, the online retail giant, provides crowdsourcing service platform, Mechanical Turk) or a third-party crowdsourcing community, such as help.zhubajie.com and InnoCentive.

1.2. Crowdsourcing Community Supply Chain. According to the role and function of the participation body in the crowdsourcing community, solving problems is the production process of a crowdsourcing community, which builds the supply chain of the crowdsourcing community, as shown in Figure 1.
In the crowdsourcing community supply chain, the outsourcer who sits upstream subcontracts some internal tasks to online users through the crowdsourcing community and offers them rewards; meanwhile, the outsourcer downstream consumes the innovative solution produced by the midstream of the supply chain named outsourcee. The outsourcee is rewarded upon completion of tasks. The crowdsourcing community is a link in the supply chain, providing the platform that connects outsourcers and outsourcees. The value of the entire supply chain rises with the participation of users.

It is apparent that the key to guaranteeing orderly operation in the entire supply chain is keeping outsourcers and outsourcees motivated so that they take a positive role in the crowdsourcing community; therefore, it is significant and practical to explore the motivation system of crowdsourcing communities.

1.3. Summary of Current Motivation Systems of Crowdsourcing Communities. The current motivation systems of crowdsourcing communities fall into three categories. In the first, the outsourcer offers fixed bonuses, which is what most crowdsourcing communities do. In the second way, the outsourcers offer such awards as patents and accumulated points according to outsourcee's efforts. Participants providing innovative ideas to the crowdsourcing platform can obtain patent grade points which can be exchanged for cash. In the third way, the crowdsourcing community offers awards; http://www.chinavalue.net/ offers one such example. This platform sends half its share to registered users, who can accumulate by joining in innovative projects, such as the number of projects they participate in, the number of times of winning the bidding, and the number of published articles, to obtain shares and bonus.

\section{Literature Review}

\subsection{Outsourcees}

2.1.1. Meeting the Psychological Needs. The research of Teresa from the Business College at Harvard and Robert J. Stenberg from Yale University showed that creative people are good self-motivators and are willing to listen to their psychological needs. Therefore, many people participate in crowdsourcing for entertainment, self-expression, self-test, and so forth [2, 3]. Qiuyan showed that people pay the most attention to aspects such as fun, self-approval, and sense of belonging in a virtual community [4]. Most participants would like 
to express their value proposition, to show their unique lifestyle, to meet their psychological needs, and to gain the conveniences the products bring them.

2.1.2. Financial Returns. Crowdsourcing is growing in popularity because people who offer creative solutions can get paid [5]. Lakhani and Wolf investigated the participants of SourceForge and found that one-third of interviewees indicated that receiving payment was their main motivation for joining SourceForge [6]. In addition, Lakhani and von Hippel investigated outsourcees who have solved tasks and received payment. The results of the investigation show that $65.8 \%$ of the interviewees have doctorate degrees and are engaging in scientific research and the main purpose of their participation is to get paid [7]. Organisciak tried to explain the participation motives of users through Maslow's hierarchy of needs and suggested that the purpose of participants is to meet the needs of society and growth. However, the investigation of 13 crowdsourcing websites showed that offering rewards can attract users to participate in crowdsourcing actively [8].

2.1.3. Learning New Knowledge and Skills. Brabham also investigated 651 participants of iStockphoto and the results showed that learning new knowledge and skills is the primary motivation for participation [9]. Through the Uses and Gratifications Theory, Brabham explained that users would like to take part in the creativity of the product because they can learn from the community and get more benefits than the manufacturer [10]. Crowdsourcing community provides a platform for like-minded people to publish their creativity freely which comprises sharing sources [11].

According to the summary and analysis of existing research, financial returns are the main motivation for crowdsourcing participants.

\subsection{Outsourcers}

2.2.1. Cost Reduction. Some researchers indicated that crowdsourcing released tasks online and did not require recruiting employees or setting up offices, which could reduce great costs. Crowdsourcing offers a way to reduce costs [12-14].

2.2.2. Making Use of Collective Wisdom. Some online users have the ability to solve specific problems. Under a similar level of knowledge and skills, the collective wisdom is better than a single wise person. A company can let volunteers from all over the world and internal staff work together to solve problems rather than forcing inner staff to learn continuously [15], which was supported by Xiao et al. who considered that, in a business economy, one problem cannot be solved by a single person but requires people with different knowledge, information, and skills. Crowdsourcing makes full use of this collective wisdom using a network of people to solve complex problems [16-18].

2.2.3. Producing Products More in Line with Consumers' Demand. Crowdsourcing is an example of the culture of mass participation; that is, universal participation of crowdsourcing enables many hobbyists to develop their talents during participation and interaction [19]. In many crowdsourcing models, users will meet the requirements of more audiences and spread the information needed by themselves [20]. This production can better meet consumer needs, can shorten the distance between the brand and the users, and can establish brand trust and strengthen customer loyalty. Using sports television programs as an example, Shi pointed out that sports game television programs are almost all the same. However, after adopting crowdsourcing, the programs have been diversified, which also stimulates the awareness of mass participation. Using news media as an example, Wei pointed out that users can participate in a news release that made "my news, I decide" come true [21].

The main purpose of outsourcers is to collect wisdom and find higher-quality solutions including better technology, more adaption to market, and lower costs.

From this discussion, we can see that a crowdsourcing community can exist and run effectively because the upstream, midstream, and downstream gains benefit from the supply chain.

\section{Model Assumption}

The motivation system design of the supply chain in a crowdsourcing community is the contract design between the outsourcer and the outsourcee; that is, the outsourcer entrusts outsourcees to finish innovative tasks through crowdsourcing community expecting to get innovative solutions with a higher quality while the outsourcees complete the tasks according to the requirements and get paid accordingly.

(1) Enterprises that release tasks are called outsourcer. Those enterprises are mutually independent and risk neutral. The outsourcer's expected utility is equal to expected profit.

(2) Users who accept tasks are called the outsourcee. These users are mutually independent.

(3) $w$ is the regular fee the outsourcer pays, $b$ refers to the incentive pay factor, and $w$ is the regular payment the outsourcee gets. The incentive pay factor is the sharing proportion of the benefits the outsourcee gets from the outsourcer.

(4) $i$ is the technical level of the outsourcee, where $i$ equal to 1 means the technical level is very high and $i$ equal to 2 means the technical level is low.

(5) $p_{i}$ is the probability the outsourcer chooses the outsourcees. $i=1$ is the high technical level outsourcee. $i=2$ is the low technical level outsourcee. Therefore, $p_{1}+p_{2}=1$.

(6) $a$ is the quality of the innovative solution offered by the outsourcee. Because the quality of the solution has a direct impact on the product or service offered by the outsourcer, it will affect income; then income is an increasing function of quality. We assume the function of the outsourcer's income is a linear function, which can be expressed as

$$
R(a)=a+\omega,
$$

where $\omega$ is the random variable from 0 to $\delta^{2}$, which is the exogenous uncertainty affecting the income. 
(7) The outsourcer can affect the outsourcee's decision by adjusting payments. We assume the payoff function is linear and can be expressed as

$$
T(R(a))=w+b R(a) .
$$

(8) Outsourcee's cost is a function of his/her innovation plan's quality and technology level, denoted by $C(a, i)$. For the outsourcee at the same level, that is, on the condition that the value of $i$ is definite, the higher the quality of the provided plan, the bigger the cost $C$. On the condition that the quality $a$ of the provided plan is definite, the marginal cost of outsourcee, whose $i=1$, is lower than that of outsourcee, whose $i=2$; according to theoretical model of supply chain analysis, set the cost function of outsourcee as $C(a, i)=\theta_{i} a^{2}$, wherein $\theta_{i}$ is outsourcers' cost coefficient, according to the difference of technology level of outsourcee, $\theta_{1}<\theta_{2}$.

(9) The maximum utility is recorded as $\bar{u}$ when the outsourcee does not perform any tasks.

According to the assumption, the expected income function of the outsourcer can be expressed as

$$
E(R(a))=E(a+\omega)=a \quad \operatorname{Var}(R(a))=\delta^{2} .
$$

The expected utility is equal to the expected income minus the payment to the outsourcee; that is,

$$
\begin{aligned}
E U & =E(R(a)-T(R(a)))=E(a+\omega-w-b R(a)) \\
& =a-w-b a .
\end{aligned}
$$

Because the outsourcee is risk averse, the expected profit of the outsourcee is equal to the expected income minus the cost of payment and risk. Urban considered the risk cost as $(1 / 2) r b^{2} \delta^{2}[22]$, where $r$ is the risk measurement of the outsourcee and $r \geq 0$. The expected income is the payment by the outsourcer, given by $w+b R(a)$. The cost of payment is $\theta a^{2}$. The expected profit can be written as

$$
\begin{aligned}
C E & =E\left(T(R(a))-C(a, i)-\frac{1}{2} r b^{2} \delta^{2}\right) \\
& =w+b a-\theta a^{2}-\frac{1}{2} r b^{2} \delta^{2} .
\end{aligned}
$$

The equivalent utility of the outsourcee is equal to the expected profit. Therefore, to motivate the outsourcee to take part in the crowdsourcing community, the design of motivation system must meet the outsourcee's participation constraint and incentive compatible constraint.

The outsourcee's participation constraint is

$$
w_{i}+b_{i} a_{i}-\theta_{i} a_{i}^{2}-\frac{1}{2} r b_{i}^{2} \delta^{2} \geq \bar{u} \quad i=1,2 .
$$

The outsourcee's incentive compatible constraint is

$$
\begin{aligned}
& w_{1}+b_{1} a_{1}-\theta_{1} a_{1}{ }^{2}-\frac{1}{2} r b_{1}^{2} \delta^{2} \\
& \geq w_{2}+b_{2} a_{2}-\theta_{2} a_{2}^{2}-\frac{1}{2} r b_{2}{ }^{2} \delta^{2} .
\end{aligned}
$$

And the maximum value is $\max C E$. Supposing $\partial C E / \partial a=0$, then

$$
a_{i}=\frac{b_{i}}{2 \theta_{i}} \quad i=1,2
$$

Based on the above analysis, the model of the motivation system is

$$
\begin{array}{ll}
\max & \sum_{i} p_{i}\left(a_{i}-w_{i}-b_{i} a_{i}\right) \quad i=1,2 \\
\text { s.t. } \quad(I R) w_{i}+b_{i} a_{i}-\theta_{i} a_{i}^{2}-\frac{1}{2} r b_{i}^{2} \delta^{2} \geq \bar{u} \quad i=1,2 \\
\text { (IC) } w_{1}+b_{1} a_{1}-\theta_{1} a_{1}^{2}-\frac{1}{2} r b_{1}^{2} \delta^{2} \\
\geq w_{2}+b_{2} a_{2}-\theta_{2} a_{2}^{2}-\frac{1}{2} r b_{2}{ }^{2} \delta^{2} & i=1,2 \\
a_{i}=\frac{b_{i}}{2 \theta_{i}} \quad i=1,2 . &
\end{array}
$$

\section{Model Analysis}

4.1. Outsourcer Adopts the Same Fixed Payment and Incentive Coefficient for All Outsourcees. Assuming the fixed payment and incentive coefficient offered by the outsourcer are $(\widehat{w}, \widehat{b})$, then the model of motivation system should be

$$
\begin{array}{ll}
\max & \sum_{i} p_{i}\left(a_{i}-\widehat{w}-\widehat{b} a_{i}\right) \quad i=1,2 \\
\text { s.t. } \quad(I R) \widehat{w}+\widehat{b} a_{i}-\theta_{i} a_{i}^{2}-\frac{1}{2} r \widehat{b}^{2} \delta^{2} \geq \bar{u} \quad i=1,2 \\
(I C) \widehat{w}+\widehat{b} a_{1}-\theta_{1} a_{1}{ }^{2}-\frac{1}{2} r \widehat{b}^{2} \delta^{2} \\
\geq \widehat{w}+\widehat{b} a_{2}-\theta_{2} a_{2}{ }^{2}-\frac{1}{2} r \widehat{b}^{2} \delta^{2} & i=1,2 \\
a_{i}=\frac{\hat{b}}{2 \theta_{i}} \quad i=1,2 . &
\end{array}
$$

Putting (13) into (10) and (11), the Lagrange equation can be built as

$$
\begin{aligned}
\Gamma= & \max \sum_{i} p_{i}\left(\frac{\widehat{b}}{2 \theta_{i}}-\widehat{w}-\widehat{b} \frac{\widehat{b}}{2 \theta_{i}}\right) \\
& +\sum_{i} \lambda_{i}\left(\widehat{w}+\widehat{b} \frac{\widehat{b}}{2 \theta_{i}}-\theta_{i}\left(\frac{\widehat{b}}{2 \theta_{i}}\right)^{2}-\frac{1}{2} r \widehat{b}^{2} \delta^{2}-\bar{u}\right) .
\end{aligned}
$$


When $\lambda_{1}=0, \lambda_{2}=1$, the optimal first-order condition can be solved as

$$
\widehat{b}=\frac{p_{1} \theta_{2}+p_{2} \theta_{1}}{2 \theta_{1} \theta_{2} r \delta^{2}+2\left(p_{1} \theta_{2}+p_{2} \theta_{1}\right)-\theta_{1}} .
$$

Putting $\widehat{b}$ into (13), we get

$$
\begin{aligned}
& \widehat{a}_{1}=\frac{p_{1} \theta_{2}+p_{2} \theta_{1}}{2 \theta_{1}\left[2 \theta_{1} \theta_{2} r \delta^{2}+2\left(p_{1} \theta_{2}+p_{2} \theta_{1}\right)-\theta_{1}\right]} \\
& \widehat{a}_{2}=\frac{p_{1} \theta_{2}+p_{2} \theta_{1}}{2 \theta_{2}\left[2 \theta_{1} \theta_{2} r \delta^{2}+2\left(p_{1} \theta_{2}+p_{2} \theta_{1}\right)-\theta_{1}\right]} .
\end{aligned}
$$

$\widehat{a}_{1}, \widehat{a}_{2}$ are the quality of innovation plan provided by outsourcee with high-level technology and outsourcee with average-level technology, when outsourcers provide the same regular compensation and incentive coefficient.

Putting the result of $\widehat{b}, \widehat{a}_{2}$ into (11), we get

$$
\widehat{\omega}=\bar{u}+\frac{\left(2 \theta_{2} r \delta^{2}-1\right)\left(p_{1} \theta_{2}+p_{2} \theta_{1}\right)^{2}}{4 \theta_{2}\left[2 \theta_{1} \theta_{2} r \delta^{2}+2\left(p_{1} \theta_{2}+p_{2} \theta_{1}\right)-\theta_{1}\right]^{2}} .
$$

Putting $\widehat{\omega}, \widehat{b}$ into (10) and (5), we get

$$
\begin{aligned}
E \widehat{U} & =\frac{\left(p_{1} \theta_{2}+p_{2} \theta_{1}\right)^{2}}{4 \theta_{1} \theta_{2}\left[2 \theta_{1} \theta_{2} r \delta^{2}+2\left(p_{1} \theta_{2}+p_{2} \theta_{1}\right)-\theta_{1}\right]}-\bar{u} \\
C \widehat{E}_{1} & =\frac{\left(p_{1} \theta_{2}+p_{2} \theta_{1}\right)^{2}\left(\theta_{2}-\theta_{1}\right)}{4 \theta_{1} \theta_{2}\left[2 \theta_{1} \theta_{2} r \delta^{2}+2\left(p_{1} \theta_{2}+p_{2} \theta_{1}\right)-\theta_{1}\right]^{2}}+\bar{u} \\
C \widehat{E}_{2} & =\bar{u} .
\end{aligned}
$$

4.2. Outsourcer Adopts Different Fixed Payment and Incentive Coefficient to All Outsourcees. Assuming the fixed payment and incentive coefficient offered by the outsourcer are $\left(w_{i}, b_{i}\right)$, $i=1,2$, then the model of motivation system is

$$
\begin{array}{ll}
\max & \sum_{i} p_{i}\left(a_{i}-w_{i}-b_{i} a_{i}\right) \quad i=1,2 \\
\text { s.t. } \quad(I R) w_{i}+b_{i} a_{i}-\theta_{i} a_{i}^{2}-\frac{1}{2} r b_{i}^{2} \delta^{2} \geq \bar{u} \quad i=1,2 \\
(I C) w_{1}+b_{1} a_{1}-\theta_{1} a_{1}^{2}-\frac{1}{2} r b_{1}^{2} \delta^{2} & \\
\geq w_{2}+b_{2} a_{2}-\theta_{2} a_{2}{ }^{2}-\frac{1}{2} r b_{2}{ }^{2} \delta^{2} & i=1,2 \\
a_{i}= & \frac{b_{i}}{2 \theta_{i}} \quad i=1,2 .
\end{array}
$$

Putting (22) into (19), (20), and (21), the Lagrange equation can be built as

$$
\begin{aligned}
\Gamma & =\max \sum_{i} p_{i}\left(\frac{b_{i}}{2 \theta_{i}}-w_{i}-\frac{b_{i}^{2}}{2 \theta_{i}}\right) \\
& +\sum_{i} \lambda_{i}\left(w_{i}+\frac{b_{i}^{2}}{4 \theta_{i}}-\frac{1}{2} r b_{i}^{2} \delta^{2}-\bar{u}\right) \\
& +\tau\left[\left(w_{1}+\frac{b_{1}^{2}}{4 \theta_{1}}-\frac{1}{2} r b_{1}^{2} \delta^{2}\right)\right. \\
& \left.-\left(w_{2}+\frac{b_{2}^{2}}{2 \theta_{2}}-\frac{\theta_{1} b_{2}^{2}}{4 \theta_{2}^{2}}-\frac{1}{2} r b_{2}^{2} \delta^{2}\right)\right] .
\end{aligned}
$$

The optimal first-order condition can be calculated as $\lambda_{1}=0$, $\lambda_{2}=1, \tau=p_{1}$ :

$$
\begin{aligned}
b_{1}= & \frac{1}{1+2 \theta_{1} r \delta^{2}} \\
b_{2}= & \frac{p_{2} \theta_{2}}{2 p_{2} \theta_{2}^{2} r \delta^{2}+\theta_{2}-p_{1} \theta_{1}} \\
w_{1}= & \bar{u}+\frac{p_{2}{ }^{2}\left(\theta_{2}-\theta_{1}\right)}{4\left(2 p_{2} \theta_{2}{ }^{2} r \delta^{2}+\theta_{2}-p_{1} \theta_{1}\right)^{2}} \\
& +\frac{2 \theta_{1} r \delta^{2}-1}{4 \theta_{1}\left(1+2 \theta_{1} r \delta_{2}\right)^{2}} \\
w_{2}= & \bar{u}+\frac{\theta_{2} p_{2}{ }^{2}\left(2 \theta_{2} r \delta^{2}-1\right)}{4\left(2 p_{2} \theta_{2}{ }^{2} r \delta^{2}+\theta^{2}-p_{1} \theta_{1}\right)^{2}} .
\end{aligned}
$$

Putting $b_{1}, b_{2}$ into (22), we get

$$
\begin{aligned}
& a_{1}=\frac{1}{2 \theta_{1}\left(1+2 \theta_{1} r \delta^{2}\right)} \\
& a_{2}=\frac{p_{2}}{4 p_{2} \theta_{2}^{2} r \delta_{2}+2 \theta_{2}-2 p_{1} \theta_{1}} .
\end{aligned}
$$

Putting $a_{1}, a_{2}, b_{1}$, and $b_{2}$ into (19) and (5), we get

$$
\begin{aligned}
& E U= \frac{2 p_{2} r \delta^{2}\left(p_{1} \theta_{2}^{2}+p_{2} \theta_{1}^{2}\right)+p_{1} \theta_{2}+p_{2} \theta_{1}-p_{1} \theta_{1}}{4 \theta_{1}\left(1+2 \theta_{1} r \delta^{2}\right)\left(2 p_{2} \theta_{2}{ }^{2} r \delta^{2}+\theta_{2}-p_{1} \theta_{1}\right)} \\
&-\bar{u} \\
& C E_{1}= \bar{u}+\frac{\left(\theta_{2}-\theta_{1}\right) p_{2}^{2}}{4\left(2 p_{2} \theta_{2}{ }^{2} r \delta^{2}+\theta_{2}-p_{1} \theta_{1}\right)^{2}} \\
& C E_{2}=\bar{u} .
\end{aligned}
$$

Proposition 1. On the condition that outsourcers provide the same regular compensation and incentive coefficient, outsourcee with high-level technology will provide higher-level innovation plan. That is, on the condition that outsourcers provide the single regular compensation, crowdsourcing community can select outsourcee with higher-level outsourcee for outsourcers. 
Comparing $\widehat{a}_{1}$ with $\widehat{a}_{2}$, we get $\widehat{a}_{1} / \widehat{a}_{2}=\theta_{2} / \theta_{1}$; since $\theta_{2}>$ $\theta_{1}$, therefore $\widehat{a}_{1}>\widehat{a}_{2}$.

Proposition 2. When the outsourcer offers a different fixed payment and incentive coefficient, the outsourcee with a higher technical level will also offer a higher-quality solution. Therefore, the crowdsourcing community can also select the outsourcee with a higher technical level when offering various systems of payment.

Because $\theta_{2}>\theta_{1}$,

$$
\begin{aligned}
a_{2} & =\frac{p_{2}}{4 p_{2} \theta_{2}{ }^{2} r \delta^{2}+2 \theta_{2}-2 p_{1} \theta_{1}} \\
& <\frac{p_{2}}{4 p_{2} \theta_{2}{ }^{2} r \delta^{2}+2 \theta_{2}-2 p_{1} \theta_{2}} .
\end{aligned}
$$

According to the assumption, $p_{1}+p_{2}=1$. Then, we get

$$
\begin{gathered}
\frac{p_{2}}{4 p_{2} \theta_{2}{ }^{2} r \delta^{2}+2 \theta_{2}-2 p_{1} \theta_{2}}=\frac{p_{2}}{2 \theta_{2}\left(2 p_{2} \theta_{2} r \delta^{2}+1-p_{1}\right)} \\
=\frac{p_{2}}{2 \theta_{2}\left(2 p_{2} \theta_{2} r \delta^{2}+p_{2}\right)}=\frac{1}{2 \theta_{2}\left(1+2 \theta_{2} r \delta^{2}\right)} \\
<\frac{1}{2 \theta_{1}\left(1+2 \theta_{1} r \delta^{2}\right)}=a_{1} .
\end{gathered}
$$

Therefore, $a_{1}>a_{2}$.

Corollary 3. No matter what kind of incentive measurement is adopted by outsourcers, the crowdsourcing community can select outsourcee with higher-level outsourcee for outsourcers. Crowdsourcing innovation can bring innovation plan with higher-tech quality for enterprises and benefit enterprises' innovation.

Proposition 4. On the condition that outsourcers provide linear variable compensation, outsourcee with high-level technology will provide higher-level innovation plan. It is in accordance with practical state. Because of the innovation plan with highertech quality provided by outsourcee, the outsourcers' income as well as outsourcee's income can be increased, so outsourcees are willing to provide innovation plan with higher quality. In fact, they are obtaining a future option income.

First, let us compare $\widehat{a}_{1}$ with $a_{1}$. Hence,

$$
\begin{aligned}
& \widehat{a}_{1}=\frac{p_{1} \theta_{2}+p_{2} \theta_{1}}{2 \theta_{1}\left[2 \theta_{1} \theta_{2} r \delta^{2}+2\left(p_{1} \theta_{2}+p_{2} \theta_{1}\right)-\theta_{1}\right]} \\
& a_{1}=\frac{1}{2 \theta_{1}\left(1+2 \theta_{1} r \delta^{2}\right)} .
\end{aligned}
$$

The result is $\widehat{a}_{1}<a_{1}$.

Second, let us compare $\widehat{a}_{2}$ with $a_{2}$. Hence,

$$
\begin{aligned}
& \widehat{a}_{2}=\frac{p_{1} \theta_{2}+p_{2} \theta_{1}}{2 \theta_{2}\left[2 \theta_{1} \theta_{2} r \delta^{2}+2\left(p_{1} \theta_{2}+p_{2} \theta_{1}\right)-\theta_{1}\right]} \\
& a_{2}=\frac{p_{2}}{4 p_{2} \theta_{2}{ }^{2} r \delta^{2}+2 \theta_{2}-2 p_{1} \theta_{1}} .
\end{aligned}
$$

When $p_{1} \theta_{2}-p_{2} \theta_{2}+p_{2} \theta_{1}>0$, the result is $\hat{a}_{2}>a_{2}$.
Corollary 5. When the outsourcer offers the differentiated payment, the outsourcee with a normal technical level will offer a lower-quality solution compared with the offer of a fixed payment. Because the outsourcee with a normal technical level is pessimistic about future earnings, he will not try his best to offer a high-quality solution.

Proposition 6. No matter what payment strategy is adopted, the expected utility of an outsourcee with high technical level will be higher than that of an outsourcee with a normal technical level. Therefore, rewarding those outsourcees with a higher technical level is an effective way for motivation.

$$
\begin{aligned}
& \text { Comparing } C \widehat{E}_{1}, C E_{1}, C \widehat{E}_{2}, C E_{2} \\
& C \widehat{E}_{1}=\frac{\left(p_{1} \theta_{2}+p_{2} \theta_{1}\right)^{2}\left(\theta_{2}-\theta_{1}\right)}{4 \theta_{1} \theta_{2}\left[2 \theta_{1} \theta_{2} r \delta^{2}+2\left(p_{1} \theta_{2}+p_{2} \theta_{1}\right)-\theta_{1}\right]^{2}}+\bar{u} \\
& C \widehat{E}_{2}=\bar{u} \\
& C E_{1}=\bar{u}+\frac{\left(\theta_{2}-\theta_{1}\right) p_{2}^{2}}{4\left(2 p_{2} \theta_{2}^{2} r \delta^{2}+\theta_{2}-p_{1} \theta_{1}\right)^{2}} \\
& C E_{2}=\bar{u} .
\end{aligned}
$$

Because $\left(p_{1} \theta_{2}+p_{2} \theta_{1}\right)^{2}\left(\theta_{2}-\theta_{1}\right) / 4 \theta_{1} \theta_{2}\left[2 \theta_{1} \theta_{2} r \delta^{2}+2\left(p_{1} \theta_{2}+\right.\right.$ $\left.\left.p_{2} \theta_{1}\right)-\theta_{1}\right]^{2}>0$, we can get $C \widehat{E}_{1}>C \widehat{E}_{2}$.

And because $\left(\theta_{2}-\theta_{1}\right) p_{2}{ }^{2} / 4\left(2 p_{2} \theta_{2}{ }^{2} r \delta^{2}+\theta_{2}-p_{1} \theta_{1}\right)^{2}>0$, then $C E_{1}>C E_{2}$.

We get $C \widehat{E}_{1}<C E_{1}, C \widehat{E}_{2}=C E_{2}$ by calculating the differences.

Proposition 7. On the condition that outsourcers provide linear variable compensation, outsourcee with high-level technology will obtain higher expected utility than that on the condition of single compensation. Outsourcee with averagelevel technology will obtain the same expected utility under two circumstances. Linear variable compensation incentive can stimulate outsourcee with high-level technology rather than outsourcee with average-level technology. Outsourcers use linear variable compensation strategy that can attract more outsourcees with high-level technology.

Proposition 8. According to Proposition 6, the outsourcer will get a higher-quality solution, which brings about increased income when adopting the diversified payment strategy. Therefore, the outsourcer can get a higher expected utility when adopting the diversified payment strategy.

Comparing $E \widehat{U}, E U$

$$
\begin{aligned}
E \widehat{U}= & \frac{\left(p_{1} \theta_{2}+p_{2} \theta_{1}\right)^{2}}{4 \theta_{1} \theta_{2}\left[2 \theta_{1} \theta_{2} r \delta^{2}+2\left(p_{1} \theta_{2}+p_{2} \theta_{1}\right)-\theta_{1}\right]}-\bar{u} \\
E U= & \frac{2 p_{2} r \delta^{2}\left(p_{1} \theta_{2}^{2}+p_{2} \theta_{1}^{2}\right)+p_{1} \theta_{2}+p_{2} \theta_{1}-p_{1} \theta_{1}}{4 \theta_{1}\left(1+2 \theta_{1} r \delta^{2}\right)\left(2 p_{2} \theta_{2}{ }^{2} r \delta^{2}+\theta_{2}-p_{1} \theta_{1}\right)} \\
& -\bar{u} .
\end{aligned}
$$




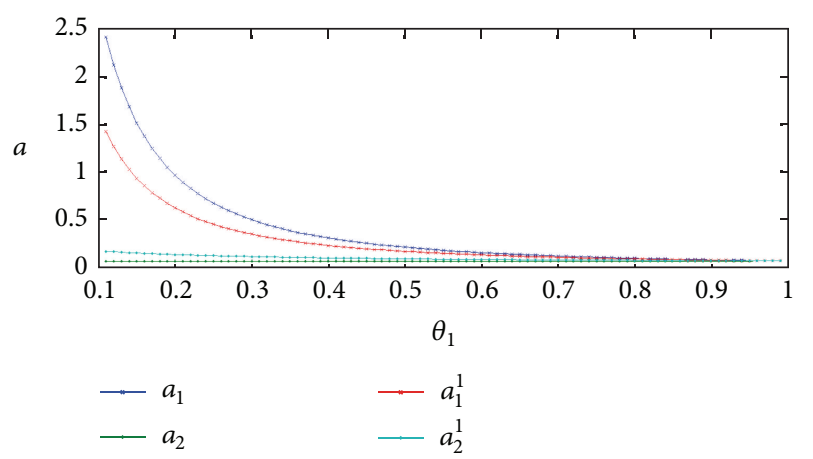

FIgURE 2: Trend of quality under various conditions.

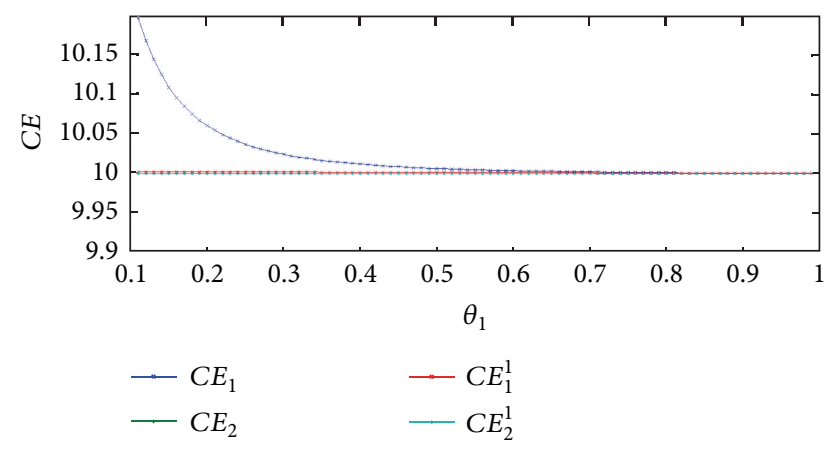

FIgURE 3: Trends of expected utilities.

4.3. Numerical Analysis. This part is to carry out example analysis in MATLAB, to concretely test the results from the models. The parameters in examples are the concrete values in the following parts. $\widehat{a}_{1}$ and $\widehat{a}_{2}$ will be represented as $a_{1}{ }^{1}$ and $a_{2}{ }^{1}$ in the MATLAB input. $C \widehat{E}_{1}$ and $C \widehat{E}_{2}$ will be represented as $C \widehat{E}_{1}{ }^{1}$ and $C \widehat{E}_{2}{ }^{1}$ in the MATLAB input.

When $\theta_{2}=1$, the abscissa is $\theta_{1}$ increasing (from 0.1 to 1 ), as shown in Figures 2 and 3.

It is shown in Figure 2 that (1) $\widehat{a}_{1}>\widehat{a}_{2}, a_{1}>a_{2}$. That is, no matter what kind of incentive mechanism is adopted, outsourcee with high-level technology can provide plan with higher quality. It is in accordance with the conclusions of Propositions 1 and 2 and Corollary 3. (2) One has $a_{1}>\widehat{a}_{1}$, $\hat{a}_{2}>a_{2}$. That is, when linear variable compensation is used, outsourcee with high-level technology can provide plan with higher quality, and outsourcee with average-level technology can provide plan with lower quality. It is in accordance with the conclusions of Proposition 4 and Corollary 5.

It is shown in Figure 3 that (1) $C \widehat{E}_{1}>C \widehat{E}_{2}, C E_{1}>C E_{2}$. No matter what kind of incentive mechanism is used, the outsourcee with high-level technology will have higher expected utility, in accordance with the conclusion of Proposition 6. (2) One has $C \widehat{E}_{1}<C E_{1}, C \widehat{E}_{2}=C E_{2}$; that is, under the linear variable compensation strategy, outsourcee with highlevel technology will obtain higher expected utility than that on the condition of single compensation, and outsourcee with average-level technology will obtain the same expected utility under two circumstances. It is in accordance with the conclusion of Proposition 7.

\section{Results and Discussion}

The model results have strong practical significance for crowdsourcing community design.

Firstly, no matter which kind of incentive mechanism is adopted, outsourcers can select the best innovation plan to improve innovation efficiency. The development of the Internet helped to develop open innovation and mass production and to release collective intelligence. Meanwhile, customers' needs become more various. Only when products sufficiently meet customers' need can they be sold on the market. Users innovation becomes the best way for enterprises to merge customers' needs. Outsourcees, as the online mass in crowdsourcing community, are the designers of innovation plans and customers. In fact, they are the users' innovation. They have a better idea about customers' needs, so the innovation plans are more attuned to customers' willingness. Therefore, crowdsourcing community can select the optimal innovation plan for enterprises. Meanwhile, because of the low cost of operating on the Internet, the selecting process is of low cost. Therefore, it is a beneficial strategy for enterprises to participate in crowdsourcing. In reality, it is the reason why the global top 500 companies actively take part in crowdsourcing.

Secondly, linear variable compensation strategy is outsourcers' better bid strategy choice. This conclusion provides basis for outsourcers' bid. In reality, there is a "lemon effect" (lemon effect: the most famous Akeerluofu thought is the so-called lemon market theory; ripe lemon is sweet, but a man says the green lemon is sweet, because he only has green lemon; this is a kind of negative psychological defense; there is a serious problem of information asymmetry between enterprises and IT enterprises; in an IT game between enterprises and enterprise customers, the final result is often a suboptimal choice, that is, the so-called "lemon effect") in crowdsourcing community innovation. Single regular compensation strategy makes online mass of outsourcees select simple crowdsourcing projects, and they are unwilling to invest more spare time in crowdsourcing projects. At the same time, because of simplifying tasks, outsourcers are unwilling to pay more, so the "lemon effect" comes into being. We need to extend the contract relationship between the outsourcer and the outsourcee. One strategy is that outsourcers provide longer-term compensation to stimulate outsourcee to participate in innovation continually. Just like continually upgrading software, outsourcers make outsourcees optimize their project design again and again.

\section{Conclusions}

This paper analyzed the motivation system of a crowdsourcing community from the perspective of the supply chain and designed a motivation system. The analysis showed that the outsourcer of an enterprise will get a higher-quality solution through the crowdsourcing community. During participation in a crowdsourcing community, the outsourcer will encourage more outsourcees with high technical level to join and force some of the outsourcees with normal technical level to quit in order to improve the quality of the solution 
as long as the outsourcer adopts a diversified payment strategy.

However, this work has at least the following limitation, which will be studied in the future. In this motivation system design, we assume the users (the outsourcees) are mutually independent. However, users influence each other by mutual learning and interaction, which will be the focus of the next study.

\section{Competing Interests}

The authors declare that there are no competing interests regarding the publication of this paper.

\section{Acknowledgments}

This work is supported by the National Natural Science Foundation of China (no. 71472172), Social Science Foundation of Sichuan (no. SC13E012), Soft Science Foundation of Sichuan (no. 2015ZR0167), and Foundation of Sichuan Library and Intelligence Research Center (no. SCTQ2016YB15).

\section{References}

[1] J. Howe, “The rise of crowdsourcing," Wired Magazine, vol. 14, no. 6, pp. 176-183, 2006.

[2] J. Howe, Crowdsourcing: Why the Power of Online Masses Drives the Future of Business, CITIC Publishing House, Beijing, China, 2009.

[3] L. Changjiang, X. Jing, and S. Fan, "Realization mechanism of mutual trust among the enterprises in supply chain nodes," Industrial Engineering and Management, no. 2, pp. 89-91, 2005.

[4] Z. Qiuyan, "Empirical research on continued participation behaviors of users in crowdsourcing community," Journal of Dalian University of Technology, no. 3, pp. 1-6, 2011.

[5] D. Ding and J. Chen, "Coordinating a three level supply chain with flexible return policies," Omega, vol. 36, no. 5, pp. 865-876, 2008.

[6] K. R. Lakhani and R. G. Wolf, "Why hackers do what they do: understanding motivation and effort in free/open source software projects," in Perspectives on Free and Open Source Software, J. Feller, B. Fitzgerald, S. Hissam, and K. R. Lakhani, Eds., no. 3, pp. 3-21, MIT Press, Cambridge, Mass, USA, 2005.

[7] K. R. Lakhani and E. von Hippel, "How open source software works: 'free' user-to-user assistance," Research Policy, vol. 32, no. 6, pp. 923-943, 2003.

[8] P. Organisciak, Why bother examining the motivations of users in large-scale crowd-powered online initiatives [M.S. thesis], University of Alberta, Master of Arts, 2010.

[9] D. Brabham, Crowdsourcing, MIT Press, 2013.

[10] D. C. Brabham, "Moving the crowd at threadless: motivations for participation in a crowdsourcing application," Information Communication \& Society, vol. 13, no. 8, pp. 1122-1145, 2010.

[11] E. Simpson and S. Roberts, "Bayesian methods for intelligent task assignment in crowdsourcing systems," in Decision Making: Uncertainty, Imperfection, Deliberation and Scalability, vol. 538, pp. 1-32, Springer, 2015.

[12] F. Liu, "The application of knowledge management in the internet witkey mode in China," Journal of Knowledge and System Sciences, vol. 4, no. 4, pp. 32-41, 2007.
[13] Q. Liping, "The path of achieving innovation coordination selected by the Collaborative Innovation Network Organization," Science \& Technology Progress and Policy, no. 5, pp. 16-19, 2011.

[14] Z. Peng and L. Ruoyu, "Research on crowdsourcing innovation motivation mechanism-based on the principal-agent theory," Technology Economics and Management Research, , no. 6, pp. 4549, 2012.

[15] L. Liang and X. Binglei, "The supervision and incentive of supply chain," Systems Engineering-Theory Methodology Applications, no. 3, pp. 21-23, 2002.

[16] X. Lan and G. Changchun, "Crowdsourcing-Changing enterprises' innovation model," Economic Research of Shanghai, no. 3, pp. 35-41, 2010.

[17] J. Prpić, P. P. Shukla, J. H. Kietzmann, and I. P. McCarthy, "How to work a crowd: developing crowd capital through crowdsourcing," Business Horizons, vol. 58, no. 1, pp. 77-85, 2015.

[18] C. Changbin and C. Gongyu, "The relationship of the supply chain on the formation and development," Industrial Technology \& Economy, vol. 25, no. 11, pp. 24-28, 2006.

[19] Z. Libin, "Research overview on problems of crowdsourcing," Science \& Technology Progress and Policy, no. 3, pp. 154-158, 2012.

[20] S. Yingjie, "The prisoners dilemma analysis of cooperation innovation in supply chain," Technology Economics and Management Research, no. 5, pp. 25-33, 2009.

[21] W. Shuancheng, "The generation and development of crowdsourcing and the path observed when building the business model of crowdsourcing," Shanghai Management Science, no. 2, pp. 55-57, 2010.

[22] G. L. Urban and E. Von Hippel, "Lead user analyses for the development of new industrial products," Management Science, vol. 34, no. 5, pp. 569-582, 1988. 


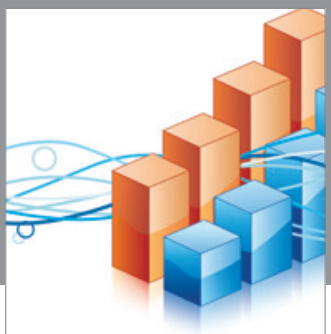

Advances in

Operations Research

vatem alat4

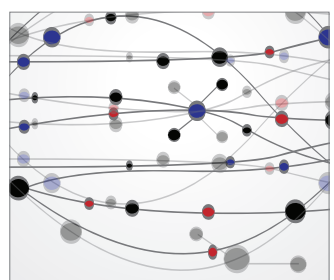

\section{The Scientific} World Journal
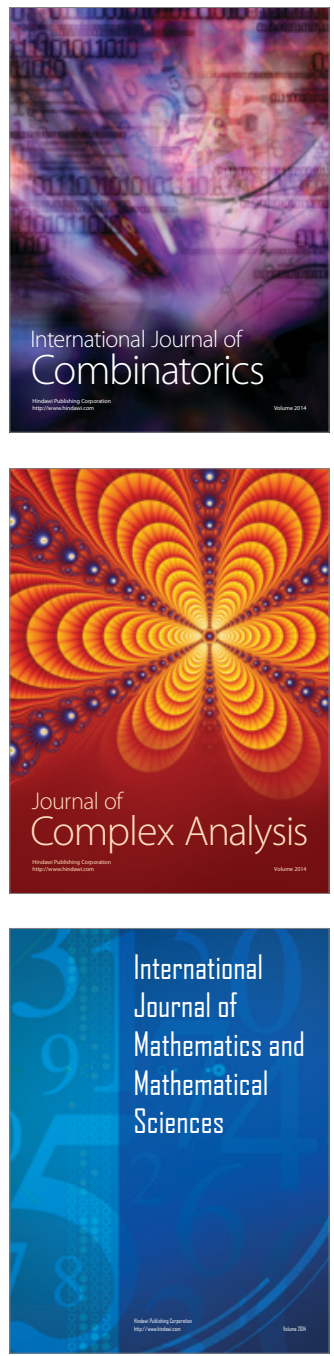
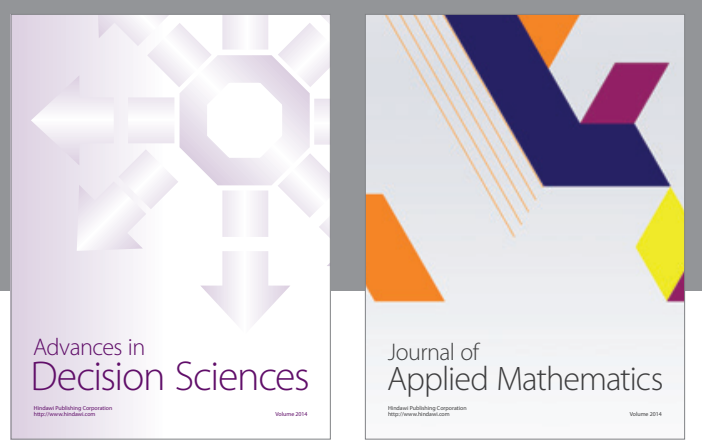

Algebra

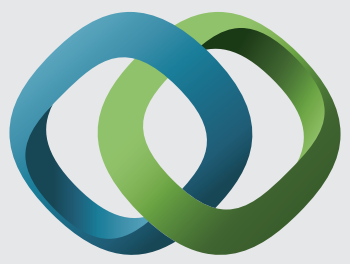

\section{Hindawi}

Submit your manuscripts at

http://www.hindawi.com
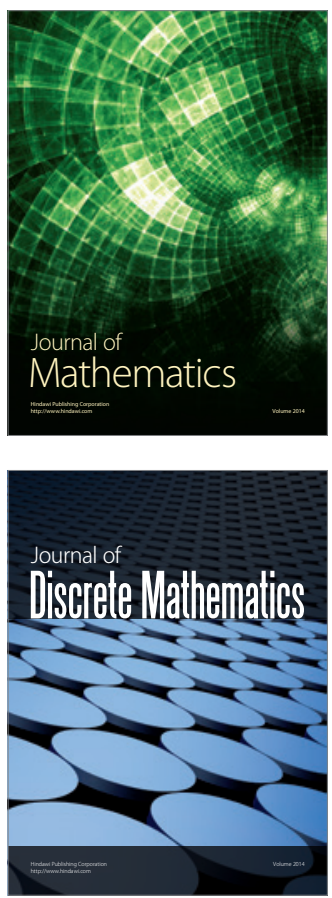

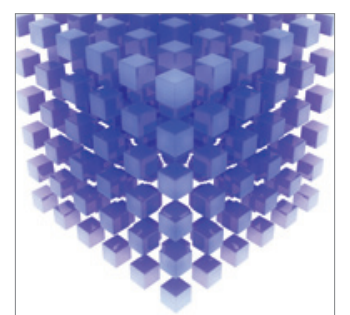

Mathematical Problems in Engineering
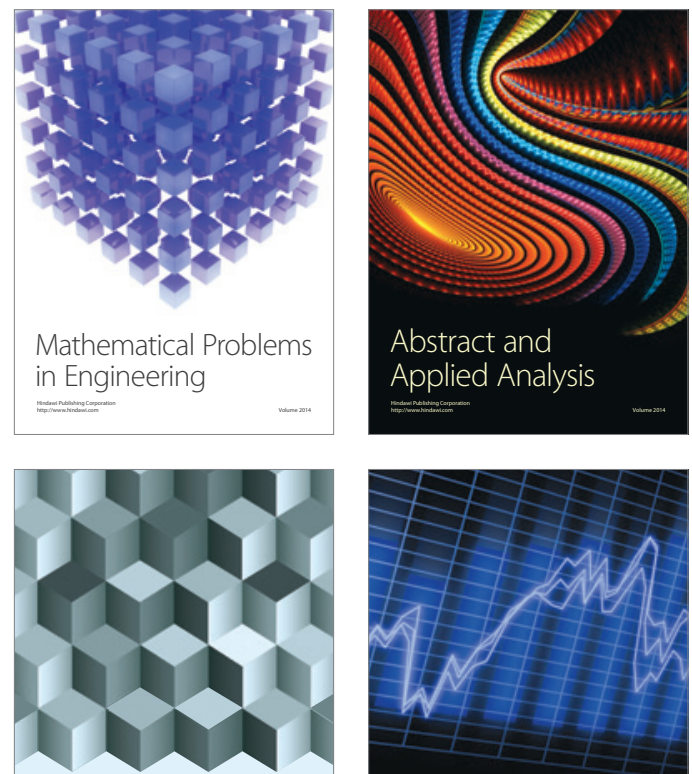

Journal of

Function Spaces

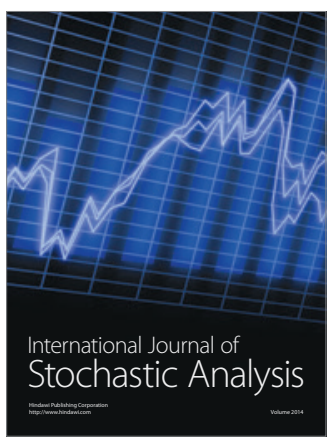

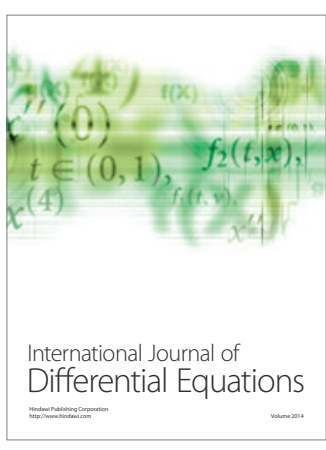
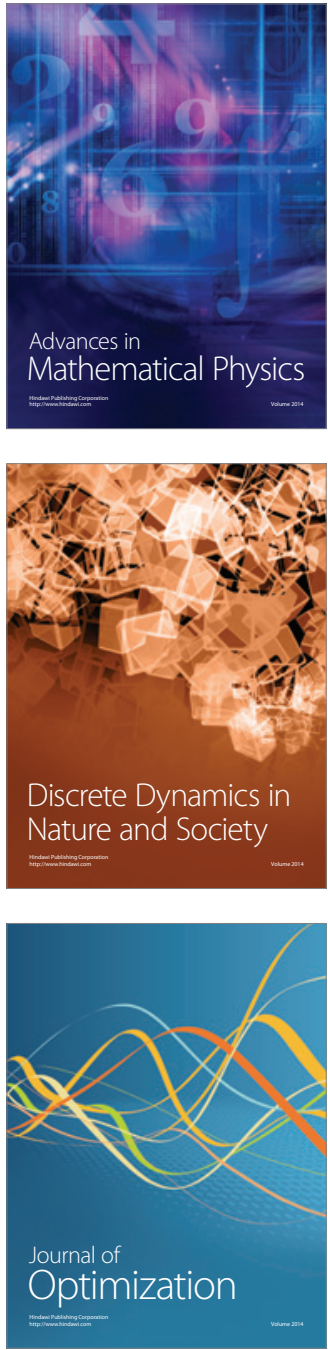